\title{
Two new Double-lined Spectroscopic Binary White Dwarfs
}

\author{
Mukremin Kilic $^{1}$, A. Bédard ${ }^{2}$, P. Bergeron ${ }^{2}$, Alekzander Kosakowski $^{1}$ \\ ${ }^{1}$ Homer L. Dodge Department of Physics and Astronomy, University of Oklahoma, 440 W. Brooks St., Norman, OK, 73019, USA \\ ${ }^{2}$ Département de Physique, Université de Montréal, C.P. 6128, Succ. Centre-Ville, Montréal, QC H3C 3J7, Canada
}

Submitted 18 February 2020

\begin{abstract}
We present radial velocity observations of four binary white dwarf candidates identified through their over-luminosity. We identify two new double-lined spectroscopic binary systems, WD 0311-649 and WD 1606+422, and constrain their orbital parameters. WD 0311-649 is a $17.7 \mathrm{~h}$ period system with a mass ratio of $1.44 \pm 0.06$ and WD $1606+422$ is a $20.1 \mathrm{~h}$ period system with a mass ratio of $1.33 \pm 0.03$. An additional object, WD 1447-190, is a $43 \mathrm{~h}$ period single-lined white dwarf binary, whereas WD 1418-088 does not show any significant velocity variations over timescales ranging from minutes to decades. We present an overview of the 14 over-luminous white dwarfs that were identified by Bédard et al., and find the fraction of double- and single-lined systems to be both $31 \%$. However, an additional $31 \%$ of these overluminous white dwarfs do not show any significant radial velocity variations. We demonstrate that these must be in long-period binaries that may be resolved by Gaia astrometry. We also discuss the over-abundance of single low-mass white dwarfs identified in the SPY survey, and suggest that some of those systems are also likely long period binary systems of more massive white dwarfs.
\end{abstract}

Key words: stars: evolution — white dwarfs — stars: individual: WD 0311-649, WD 1418-088, WD 1447-190, WD 1606+422

\section{INTRODUCTION}

Double white dwarfs with well-measured orbital and physical parameters provide essential constraints on binary population synthesis models and the stability of mass transfer in their progenitor systems. However, the number of double white dwarfs with well measured primary and secondary masses and orbital parameters is rather small, with only 8 eclipsing and 17 double-lined (SB2) spectroscopic binaries currently known.

The eclipsing systems are dominated by extremely low-mass (ELM, $M \sim 0.2 M_{\odot}$ ) white dwarfs (e.g., Steinfadt, et al. 2010; Brown, et al. 2011, 2017; Burdge, et al. 2019). This is not surprising, as the eclipse searches are most sensitive to short period systems, and the formation of ELM white dwarfs requires close binary companions ( $\mathrm{Li}$, et al. 2019). ELM white dwarfs tend to have relatively massive companions (Andrews, Price-Whelan \& Agüeros 2014; Boffin 2015; Brown, et al. 2016), and six of the known eclipsing systems have relatively large mass ratios. The two exceptions are the low-mass white dwarfs CSS $41177\left(0.38+0.32 M_{\odot}\right.$, Bours, et al. 2014) and $\mathrm{J} 1152+0248\left(0.47+0.44 M_{\odot}\right.$, Hallakoun, et al. 2016).

Double-lined spectroscopic binaries, on the other hand, are biased towards equal brightness and equal mass systems. The first SB2 white dwarf system, L870-2, was identified as a potential binary based on its over-luminosity compared to other white dwarfs, and was confirmed to be a double-lined spectroscopic binary by Saffer, Liebert \& Olszewski (1988). Radial velocity surveys in the late 1990s and early 2000s, mainly the ESO supernovae type Ia progenitor survey (SPY, Napiwotzki, et al. 2001), increased the number of SB2 systems to 13 (Marsh 1995; Moran, Marsh \& Bragaglia 1997; Maxted, Marsh \& Moran 2002; Napiwotzki, et al. 2002; Karl, et al. 2003; Napiwotzki, et al. 2007). With the serendipitious discovery of an additional system by Debes, et al. (2015), and three more by Rebassa-Mansergas, et al. (2017), the total number of double-lined WDs with well-measured orbital parameters and mass ratios is now 17. There are nine more SB2 systems identified by Napiwotzki, et al. (2019) that need follow-up radial velocity observations for orbital constraints.

Recently, Bédard, Bergeron \& Fontaine (2017) performed a spectroscopic and photometric analysis of 219 white dwarfs with trigonometric parallax measurements available at the time, and identified 15 unresolved double degenerate binary candidates, including several previously known double-lined spectroscopic binaries. Bergeron, et al. (2019) presented an updated analysis of these objects based on Gaia Data Release 2 parallaxes, and confirmed the overluminous nature of all but one of these targets, WD1130+189. Here we present follow-up spectroscopy of four of these white dwarfs, three of which are confirmed to be binary systems. We present an overview of the over-luminous white dwarf sample from Bédard, Bergeron \& Fontaine (2017) and discuss the period and mass distribution of the sample, as well as the fractions of SB2, SB1, and systems that show no significant radial velocity variability. 

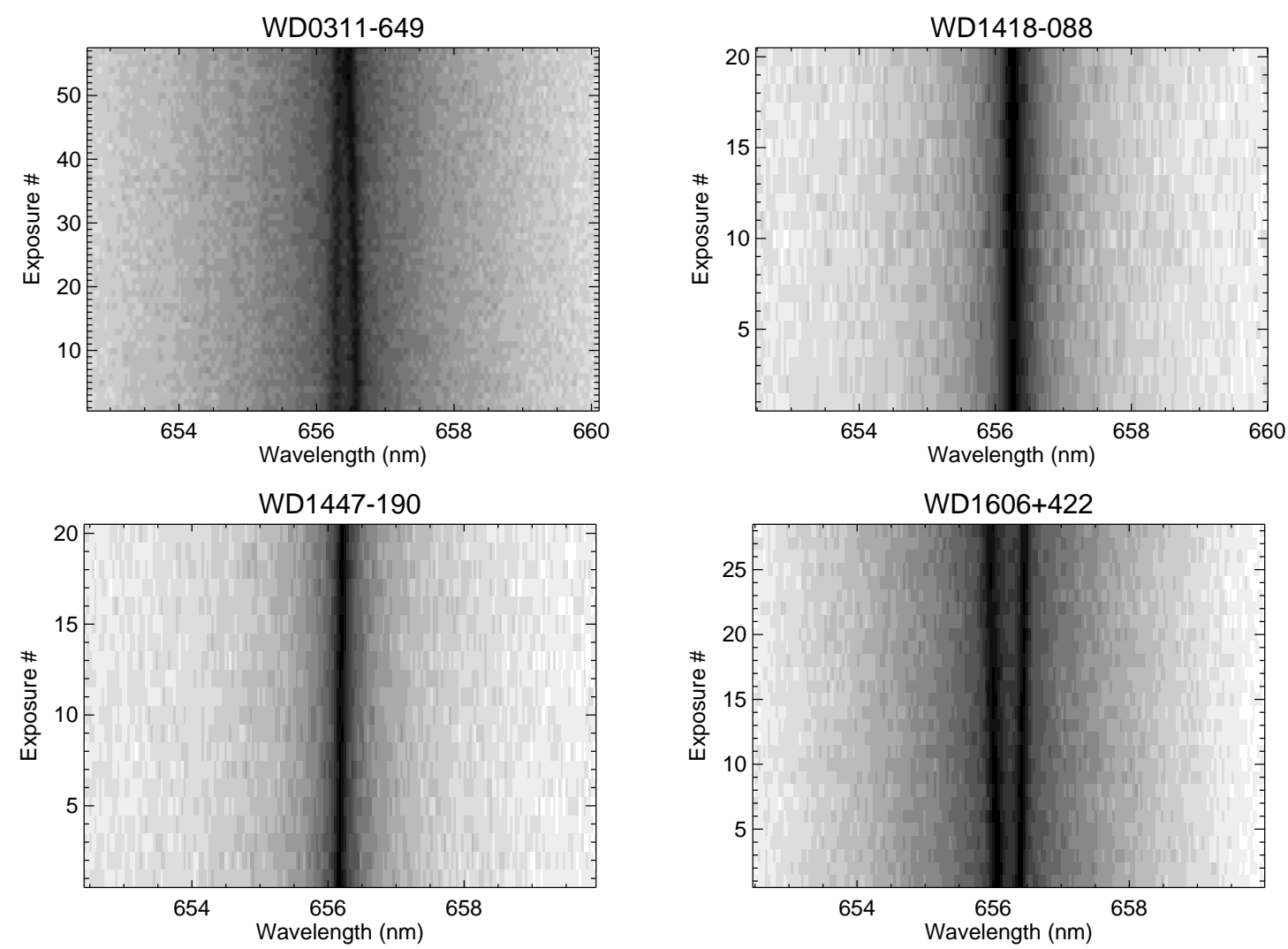

Figure 1. Gemini time-resolved spectroscopy of four white dwarfs with back-to-back exposures. WD 0311-649 and WD 1606+422 turn out to be double-lined spectroscopic binaries, whereas WD 1447-190 is a single-lined binary. The remaining target, WD 1418-088, does not show any significant radial velocity variability.

\section{OBSERVATIONS}

We used the HIRES echelle spectrometer (Vogt et al. 1994) on the Keck I telescope at Mauna Kea Observatory in Hawaii to observe three of our targets on UT 2018 June 18. Unfortunately our three half-night long observing run was limited to a period of about only 2 hours due to volcanic activity and vog. We used the blue cross disperser with a 1.15 arcsec slit resulting in a spectral resolution of 37,000. We used MAKEE to analyze the HIRES data.

We obtained follow-up optical spectroscopy of all four of our targets using the $8 \mathrm{~m}$ Gemini telescopes equipped with the Gemini Multi-Object Spectrograph (GMOS) as part of the queue programs GN-2018A-Q-116 and GS-2018B-Q-117. We used the R831 grating and a $0.25^{\prime \prime}$ slit, providing wavelength coverage from $5380 \AA$ to $7740 \AA$ and a resolution of $0.376 \AA$ per pixel. Each spectrum has a comparison lamp exposure taken within $10 \mathrm{~min}$ of the observation time. We used the IRAF GMOS package to reduce these data.

Our initial observing strategy included a series of back-toback exposures to look for short period systems. Figure 1 shows the Gemini/GMOS trailed spectra for all four targets based on these back-to-back exposures. We obtained $57 \times 150 \mathrm{~s}$ exposures of WD 0311-649 on UT 2018 Oct $2,20 \times 300$ s exposures of WD 1418-088 on UT 2018 July $1,20 \times 300 \mathrm{~s}$ exposures of WD 1447-190 on UT 2018 July 10 , and $28 \times 245$ s exposures of WD $1606+422$ on UT 2018 Sep 11. This figure reveals two double-lined binary systems, WD 0311-649 and WD 1606+422, where the double $\mathrm{H} \alpha$ lines are seen converging and diverging over a few hours, respectively. One of the single-lined objects, WD 1447-190, also showed significant velocity shifts in the back-to-back exposures, but the other, WD
1418-088, did not show any significant variations over a period of $1.8 \mathrm{~h}$, and we decided not to follow it up further (see more below). To constrain the orbital parameters of the three velocity variable systems, we obtained additional spectroscopy with different nightly cadences as part of the Gemini Fast Turnaround and queue programs GN-2019A-FT-208, GS-2019A-FT-202, and GS-2019B-Q-113.

We obtained seven additional spectra of WD 1447-190 on UT 2019 March 1-3 at the 4.1m SOAR telescope equipped with the Goodman High Throughput spectrograph (Clemens, Crain \& Anderson 2004) with the 930 line $\mathrm{mm}^{-1}$ grating and the $1.03^{\prime \prime}$ slit. This set-up provides $2.2 \AA$ spectral resolution over the range 3550 - $5250 \AA$. The SOAR spectra were obtained as part of the NOAO program 2019A-0134.

\section{RADIAL VELOCITY MEASUREMENTS}

We use the core of the $\mathrm{H} \alpha$ line to measure the radial velocities of our single- and double-lined systems. After normalizing the continuum, we use a quadratic polynomial plus a Lorentzian or Voigt profile to fit the line wings and the line cores, respectively. We find the bestfit parameters with LMFIT, a version of the Levenberg-Marquardt algorithm adapted for Python (Newville, et al. 2014). We apply the standard Solar System barycentric corrections, and use the night skylines to check the spectrograph flexure.

Figure 2 shows the best-fit to the first Gemini exposure on the double-lined system WD 0311-649, demonstrating our procedure. Here the dotted blue and green lines show the best-fitting Lorentzian 


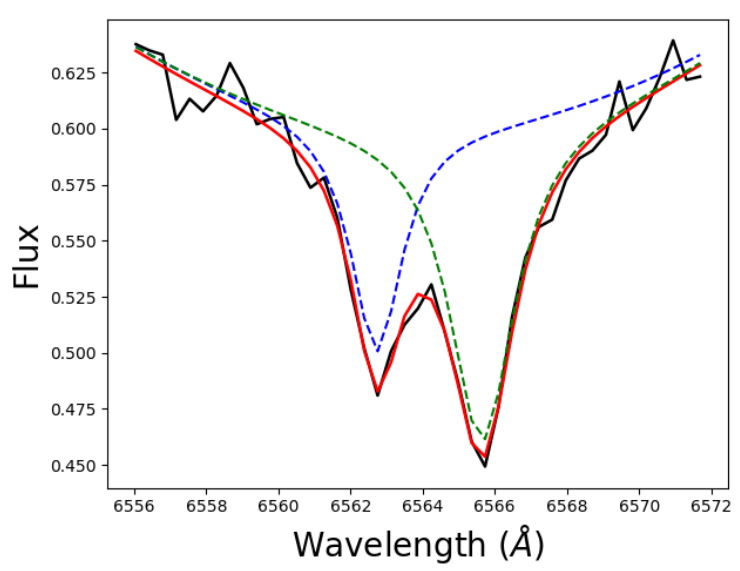

Figure 2. Best-fitting Lorentzian profiles to the $\mathrm{H} \alpha$ line cores visible in the double-lined spectroscopic binary WD 0311-649 (blue and green dotted lines). The red solid line shows the composite best-fitting model.

profiles to the two $\mathrm{H} \alpha$ line cores, and the red line shows the composite best-fitting model. The formal measurement errors on the two $\mathrm{H} \alpha$ line centers in this spectrum are 0.06 and $0.05 \AA$, respectively. Napiwotzki, et al. (2019) demonstrated that formal fitting errors tend to be underestimated, and that error estimates based on bootstrapping are better for including uncertainties from imperfections of the input data and non-Gaussian noise. We use a similar bootstrapping procedure, and randomly select $N$ points of the observed spectra, where points can be selected more than once. We use the bootstrapped spectra to rederive velocities, repeating this procedure 1000 times. We add the standard deviation of the velocity measurements from the bootstrapped spectra and the formal fitting errors in quadrature to estimate the total errors in each velocity measurement. This procedure gives $4 \mathrm{~km} \mathrm{~s}^{-1}$ errors for both lines in the spectrum shown in Figure 2.

\section{ATMOSPHERIC PARAMETER DETERMINATION}

Bédard, Bergeron \& Fontaine (2017) showed that it is possible to constrain the atmospheric parameters $\left(T_{\text {eff }}\right.$ and $\log g$ ) of both white dwarfs in an unresolved DA+DA binary system by combining spectroscopic, photometric, and astrometric information. More specifically, they developed a deconvolution procedure that involves fitting simultaneously the observed Balmer lines and spectral energy distribution with composite model atmospheres. We briefly describe this method here, as we apply it in Section 5 to revisit the atmospheric properties of our four binary candidates in light of our new data.

The radiative flux $f_{v}$ received at Earth from an unresolved double degenerate system is simply the sum of the Eddington fluxes emitted by the individual components, properly weighted by their respective solid angle:

$f_{v}=4 \pi\left(\frac{R_{1}}{D}\right)^{2} H_{v, 1}+4 \pi\left(\frac{R_{2}}{D}\right)^{2} H_{v, 2}$.

Assuming that the distance $D$ is known from a trigonometric parallax measurement, the right-hand side of this equation depends only on the four atmospheric parameters $T_{\mathrm{eff}, 1}, \log g_{1}, T_{\mathrm{eff}, 2}$, and $\log g_{2}$. Indeed, for given values of these quantities, the Eddington fluxes $H_{v, 1}$ and $H_{v, 2}$ are obtained from model atmospheres, and the radii $R_{1}$ and $R_{2}$ are obtained from evolutionary sequences. In

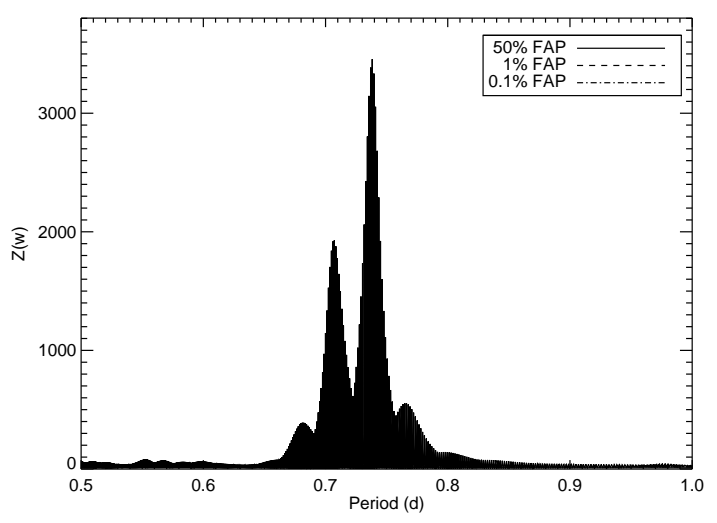

WD $0311-649$


Figure 3. Top: Lomb-Scargle periodogram for WD 0311-649. Middle and Bottom: Radial velocity measurements (open and filled points) and the bestfitting orbital solutions (dotted and solid lines) for the two stars in WD 0311-649 assuming a circular orbit.

what follows, we use pure-hydrogen model atmospheres similar to those described in Tremblay \& Bergeron (2009) together with 3D hydrodynamical corrections from Tremblay, et al. (2013), as well as evolutionary models similar to those described in Fontaine, Brassard $\&$ Bergeron (2001) with carbon/oxygen cores $\left(X_{C}=X_{O}=0.5\right)$ and standard "thick" hydrogen layers $\left(M_{\mathrm{H}} / M_{\star}=10^{-4}\right)$.

Both our spectroscopic and photometric analyses are based on Equation 1. In the spectroscopic case, the observed optical spectrum (left-hand side) is compared to a weighted sum of two synthetic spectra (right-hand side). Since only the shape of the Balmer lines is of interest, the observed and (combined) synthetic spectra are normalized to a continuum set to unity before the comparison is carried out. In the photometric case, a set of average fluxes measured in some optical and infrared bandpasses (left-hand side) is compared to a weighted sum of two synthetic spectra properly averaged over the corresponding bandpass filters (right-hand side). We adopt the zero points given in Holberg \& Bergeron (2006) to convert observed magnitudes into average fluxes, and neglect reddening since all of our systems are within $50 \mathrm{pc}$ (see Table 1). Note that absolute fluxes are required here since we are interested in the overall energy distribution.

Our fitting procedure uses the Levenberg-Marquardt algorithm to find the values of $T_{\mathrm{eff}, 1}, \log g_{1}, T_{\mathrm{eff}, 2}$, and $\log g_{2}$ that minimize the 
difference between the two sides of the equation for both the spectroscopic and photometric observations simultaneously. We stress that such a unified approach is mandatory to achieve reliable results, especially when all four atmospheric parameters are allowed to vary. We do not fit for distance, since distances are precisely determined by Gaia DR2 (Gaia Collaboration, et al. 2018) for all four targets (Table 1).

In our analysis reported below, we employ the same spectroscopic and photometric data as in Bédard, Bergeron \& Fontaine (2017), with the exceptions that we make use of our new SOAR spectrum for WD 1447-190, and that we add the Pan-STARRS grizy magnitudes (Chambers, et al. 2016) to our photometric fits for WD 1418-088, WD 1447-190, and WD 1606+422.

\section{RESULTS}

\subsection{WD 0311-649}

Figure 3 shows the radial velocity measurements and the LombScargle periodogram for the double-lined spectroscopic binary WD 0311-649. We use the IDL program MPRVFIT (De Lee et al. 2013) in the SB2 mode to find the best-fitting orbit. Excluding the three spectra where the $\mathrm{H} \alpha$ lines from both stars overlap and appear as a single line, we have 68 radial velocity measurements for each star. The best-fitting orbital parameters and their formal errors are $P=0.73957 \pm 0.00001 \mathrm{~d}, K_{1}=86.5 \pm 1.7 \mathrm{~km} \mathrm{~s}^{-1}$, $K_{2}=60.0 \pm 2.1 \mathrm{~km} \mathrm{~s}^{-1}, \gamma_{1}=48.7 \pm 1.7 \mathrm{~km} \mathrm{~s}^{-1}$, and a velocity offset of $\gamma_{2}-\gamma_{1}=11.1 \pm 2.7 \mathrm{~km} \mathrm{~s}^{-1}$.

The Lomb-Scargle diagram shows that there are significant aliases, which are offset from each other by multiples of 0.00135 d. Period aliases are the largest source of uncertainty in our orbital solutions. To constrain the impact of these aliases on our orbital solutions, we use a Monte-Carlo approach, re-sampling the radial velocities with their errors and re-fitting orbital parameters 1,000 times. This approach samples $\chi^{2}$ space in a self-consistent way. We report the median value and errors derived from the $15.9 \%$ and $84.1 \%$ percentiles of the distributions for each orbital element. The best-fitting orbital parameters from the Monte Carlo simulations are $P=0.73956_{-0.00267}^{+0.00134} \mathrm{~d}, K_{1}=86.5_{-1.7}^{+2.0} \mathrm{~km} \mathrm{~s}^{-1}, K_{2}=60.1_{-2.1}^{+2.0}$ $\mathrm{km} \mathrm{s}^{-1}, \gamma_{1}=48.4 \pm 1.7 \mathrm{~km} \mathrm{~s}^{-1}, \gamma_{2}-\gamma_{1}=11.6_{-2.7}^{+2.6} \mathrm{~km} \mathrm{~s}^{-1}$, and $\frac{K_{1}}{K_{2}}=1.44 \pm 0.06$. These values are consistent with the formal estimates from MPRVFIT within the errors, though the error in period is significantly larger than the formal errors due to the aliasing present.

The individual masses of the two components can be derived from the orbital parameters. Since the difference in systemic velocities is equal to the difference in gravitational redshifts, we have:

$$
\begin{aligned}
\gamma_{2}-\gamma_{1} & =\frac{G}{c}\left(\frac{M_{2}}{R_{2}\left(M_{2}\right)}-\frac{M_{1}}{R_{1}\left(M_{1}\right)}\right) \\
& =\frac{G}{c}\left(\frac{K_{1} M_{1} / K_{2}}{R_{2}\left(K_{1} M_{1} / K_{2}\right)}-\frac{M_{1}}{R_{1}\left(M_{1}\right)}\right)
\end{aligned}
$$

where $R(M)$ is the mass-radius relation obtained from our evolutionary sequences. For given values of $\gamma_{2}-\gamma_{1}$ and $K_{1} / K_{2}$, this equation can be solved numerically for $M_{1}$ (and hence $M_{2}$ ). We find $M_{1}=0.385_{-0.063}^{+0.060} M_{\odot}$ and $M_{2}=0.554_{-0.082}^{+0.073} M_{\odot}$.

Figure 4 displays our best model-atmosphere fit to the Balmer lines and the spectral energy distribution of WD 0311-649. In the minimization procedure, the surface gravities are held fixed to the values derived from the orbital solution, $\log g_{1}=7.55_{-0.17}^{+0.14}$ and $\log g_{2}=7.91_{-0.16}^{+0.12}$, so only the effective temperatures are treated as free parameters. Our fitting method yields $T_{\mathrm{eff}, 1}=12,600 \pm 500 \mathrm{~K}$ and $T_{\text {eff, } 2}=12,300 \pm 500 \mathrm{~K}$. Both the spectroscopic and photometric data are nicely reproduced by our composite model.

To validate our solution further, we compare in Figure 5 the observed and predicted $\mathrm{H} \alpha$ features of WD 0311-649. Five of our Gemini spectra are co-added in order to increase the signal-to-noise ratio, and a wavelength shift is applied to the individual synthetic spectra to match the observed shift between the two line cores. The agreement is almost perfect, even though no fit was performed here, and thus confirms the accuracy of our atmospheric parameters. Finally, it is interesting that our solution places the secondary star within the ZZCeti instability strip, close to the blue edge (Gianninas, Bergeron \& Ruiz 2011). Therefore, this object should be monitored for pulsations, which might however be difficult to detect due to the light of the primary star.

\subsection{WD 1418-088}

Figure 6 shows the radial velocity measurements for WD 1418-088 from the SPY survey (top panel, Napiwotzki, et al. 2019) and our Keck (middle panel) and Gemini (bottom panel) observations. WD 1418-088 does not show any significant radial velocity variations. We use the weighted mean velocity to calculate the $\chi^{2}$ statistic for a constant velocity model. The probability, $p$, of obtaining the observed value of $\chi^{2}$ or higher from random fluctuations of a constant velocity, taking into account the appropriate number of degrees of freedom is 0.22 . Hence, the null hypothesis cannot be rejected; the radial velocity measurements for WD $1418-088$ are consistent with a constant velocity.

Bédard, Bergeron \& Fontaine (2017) showed that there is a significant discrepancy between the spectroscopic and photometric solutions for WD 1418-088. Under the assumption of a single star, the spectroscopic fits (corrected for 3D effects) indicate $T_{\text {eff }}=8060$ $\mathrm{K}, \log g=8.1$, and $M=0.66 M_{\odot}$, whereas the photometric fits using the Gaia DR2 parallax measurement indicate much lower $\log g=7.55$ and $M=0.36 M_{\odot}$ (Blouin, et al. 2019). The only way to resolve the discrepancy between the photometric and spectroscopic solutions is if WD 1418-088 is a binary system. The combined light from the two stars in an unresolved binary would make it appear more luminous, which could be interpreted as a single white dwarf having a larger radius, and therefore a lower mass.

Given the limited number of radial velocity observations over monthly and yearly timescales, our observations of WD 1418-088 are not sensitive to long period systems. To estimate the detection efficiency of a binary system as a function of orbital period, we use a Monte Carlo approach, and generate synthetic radial velocity measurements with the same temporal sampling and accuracy as the WD 1418-088 observations. We assume a mass ratio of one, and include the projection effects due to randomly oriented orbits. We estimate our detection efficiency using the number of trials which satisfy the detection criterion of $\log (p)<-4$ (see Maxted et al. 2000). Figure 7 shows the detection efficiency of our observations for WD 1418-088 for orbital periods ranging from 1 to 1000 days. This figure shows that we would have detected the majority of the binary systems with orbital periods $\leqslant 70$ days, but our detection efficiency significantly deteriorates beyond 80 days. Hence, WD $1418-088$ is likely a long period binary white dwarf system. One of the over-luminous white dwarfs included in the Bédard, Bergeron \& Fontaine (2017) study, WD $1639+153$, is an astrometric binary 

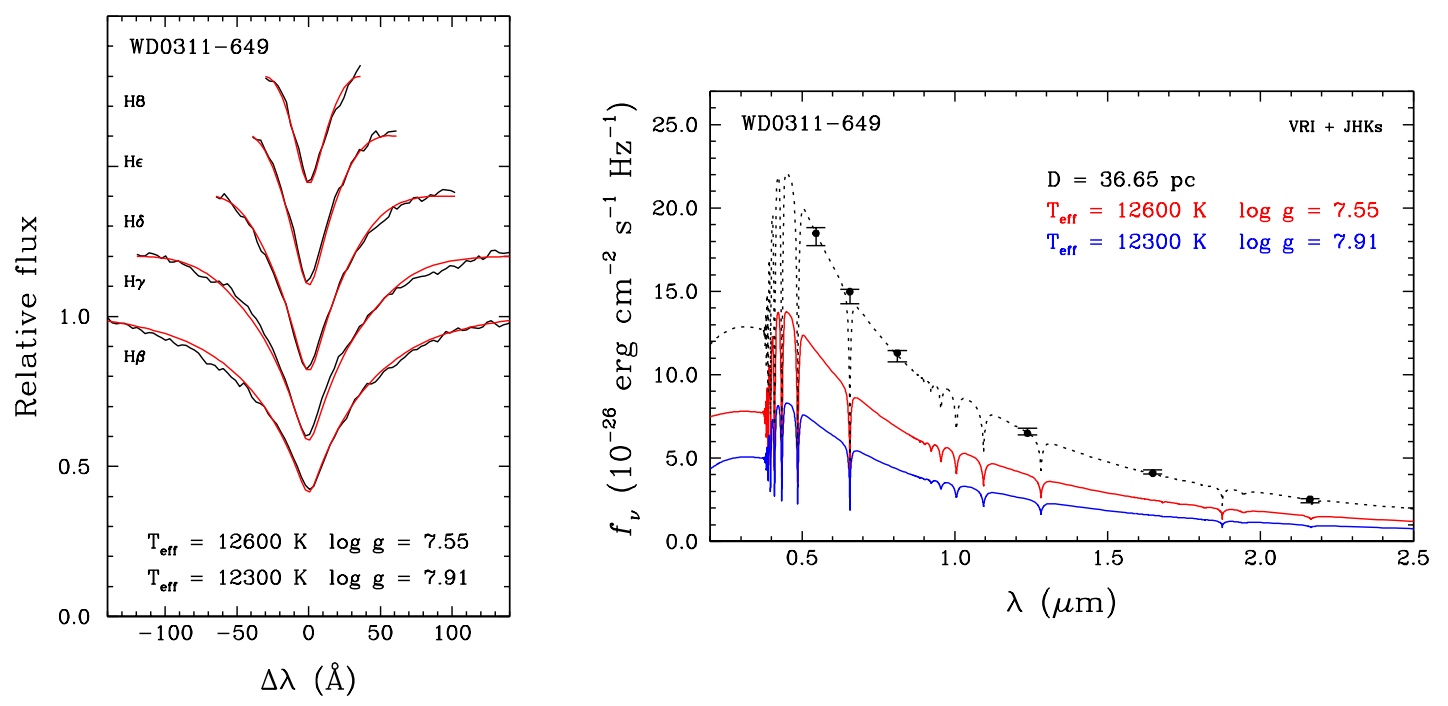

Figure 4. Best model-atmosphere fit to the Balmer lines (left panel) and the spectral energy distribution (right panel) of WD 0311-649. In the left panel, the observed and synthetic spectra are displayed as the black and red lines, respectively. In the right panel, the observed and synthetic average fluxes are shown as the error bars and filled circles, respectively; in addition, the red and blue lines show the contribution of each component to the total monochromatic model flux, which is displayed as the black dotted line. The best-fitting atmospheric parameters are given in both panels.

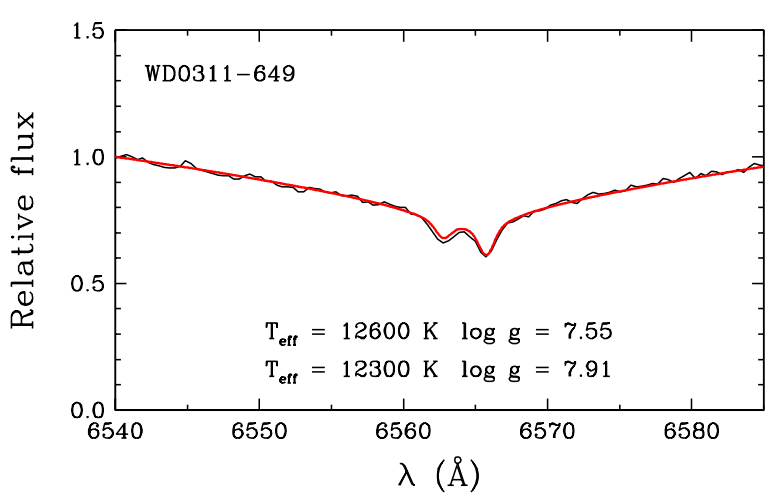

Figure 5. Comparison of the observed double H $\alpha$ feature of WD 0311-649, shown as the black line, with that predicted by our best model-atmosphere fit, displayed as the red line.

with an orbital period of 4 years. Hence, WD 1418-088 may be an unresolved binary with a similarly long orbital period.

Figure 8 displays our best model-atmosphere fit to the spectroscopic and photometric observations of WD 1418-088 (as well as WD 1447-190 and WD 1606+422) assuming a DA+DA binary system. Here, in the absence of an orbital solution, all four atmospheric parameters are allowed to vary in the fitting procedure and are thus less tightly constrained than in the case of WD 0311-649. We derive $T_{\text {eff, } 1}=8500 \pm 1000 \mathrm{~K}, \log g_{1}=8.00 \pm 0.20$ and $T_{\text {eff }, 2}=6100 \pm 500$ $\mathrm{K}, \log g_{2}=8.15 \pm 0.20$, which correspond to $M_{1}=0.60_{-0.11}^{+0.12} M_{\odot}$ and $M_{2}=0.68_{-0.12}^{+0.13} M_{\odot}$ according to the mass-radius relation. The agreement between the data and the model is excellent, but the errors in our surface gravity and mass estimates are relatively large due to the lack of any orbital constraints in this system. The best-fit model indicates a visible/near-infrared flux ratio of $\sim 2-5$ between the primary and secondary stars. If this is a long period binary,
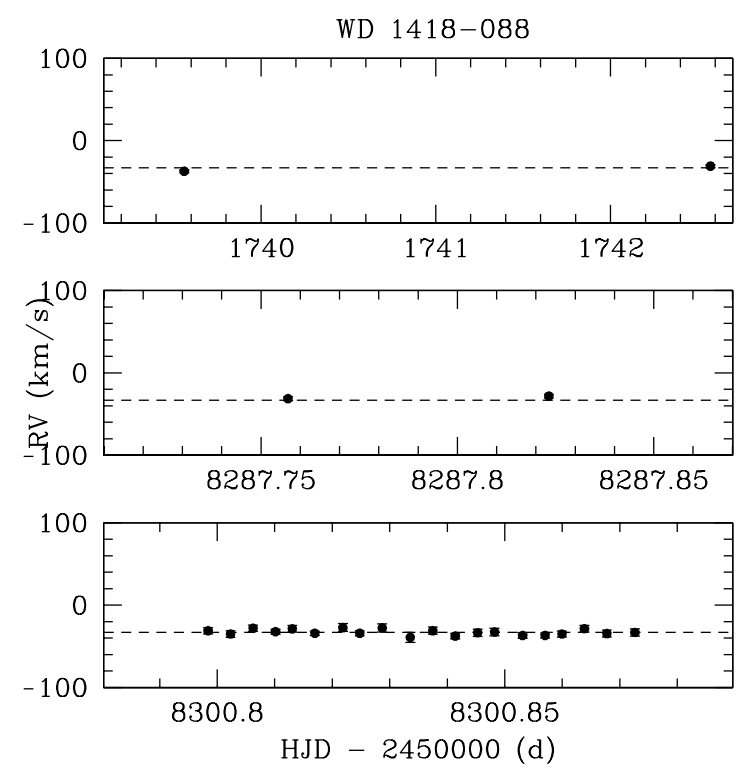

Figure 6. VLT (top), Keck (middle), and Gemini (bottom) radial velocity observations of WD 1418-088. The dotted line marks the weighted mean of the velocity measurements.

high resolution imaging observations may be able to resolve it (e.g., Harris, et al. 2013).

Andrews, Breivik \& Chatterjee (2019) demonstrate that Gaia astrometry can find hidden white dwarf companions at distances as far as several hundred parsecs. In addition, Gaia can characterize orbits with periods ranging from 10 days to thousands of days. Using 0.1 mas as the size of the primary star's orbit resolvable by Gaia (Andrews, Breivik \& Chatterjee 2019) and a distance of 37 pc, Gaia should be able to easily resolve the astrometric orbit for WD 1418-088 within the next several years. 


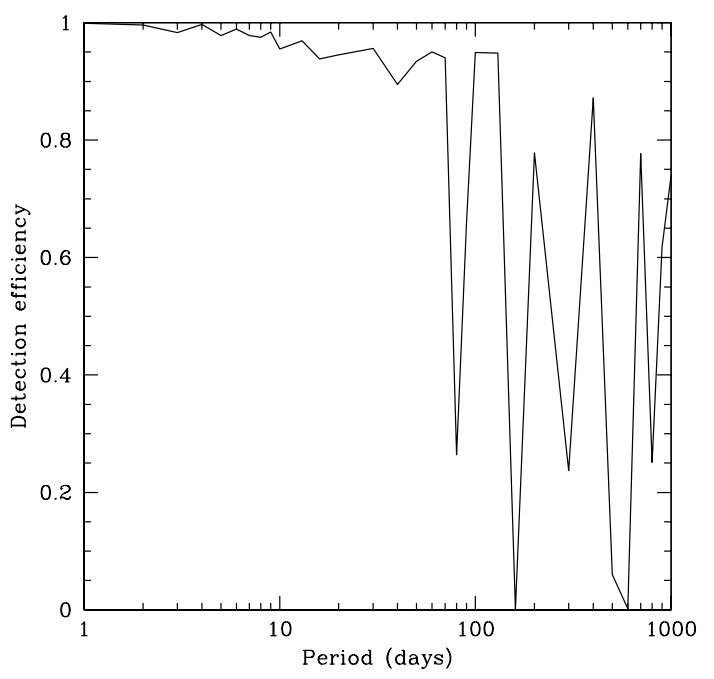

Figure 7. The detection efficiency as a function of orbital period for WD 1418-088, assuming an equal mass binary system.

\subsection{WD 1447-190}

Figure 9 shows the radial velocity measurements and the LombScargle periodogram for the single-lined binary WD 1447-190. There are no significant period aliases in the Lomb-Scargle diagram. We perform 1000 Monte Carlo simulations for our orbital fits, and the results are consistent with the formal estimates from MPRVFIT. The best-fit orbital solution has $P=1.79083 \pm 0.00004 \mathrm{~d}, K=$ $83.8_{-1.3}^{+1.2} \mathrm{~km} \mathrm{~s}^{-1}, \gamma=-32.7_{-0.9}^{+0.8} \mathrm{~km} \mathrm{~s}^{-1}$, and a mass function of $f=0.109 \pm 0.005 M_{\odot}$.

Our best model-atmosphere fit to the Balmer lines and energy distribution of WD 1447-190 is presented in Figure 8. Given the lack of a complete orbital solution, all four atmospheric parameters are considered as free parameters in the minimization process. We find $T_{\text {eff, } 1}=8000 \pm 1000 \mathrm{~K}, \log g_{1}=7.65 \pm 0.20$ and $T_{\text {eff, } 2}=5000 \pm$ $500 \mathrm{~K}, \log g_{2}=7.50 \pm 0.20$, which convert to $M_{1}=0.41_{-0.08}^{+0.10} M_{\odot}$ and $M_{2}=0.33_{-0.07}^{+0.09} M_{\odot}$ using our evolutionary sequences. Again, our composite model reproduces the spectroscopy and photometry relatively well. For $M_{1}=0.41 M_{\odot}$, the mass function requires $M_{2} \geqslant$ $0.42 M_{\odot}$, which suggests that the secondary mass is closer to the upper limit of the mass range indicated by our model atmosphere analysis. Interestingly, our solution provides an elegant explanation for the single-lined nature of this binary system: the secondary white dwarf has a very low effective temperature, hence its $\mathrm{H} \alpha$ feature is simply too weak to be observed.

\subsection{WD $1606+422$}

Figure 10 shows the radial velocity measurements and the LombScargle periodogram for the double-lined spectroscopic binary WD $1606+422$. Excluding the two spectra where the $\mathrm{H} \alpha$ lines from both stars overlap and appear as a single line, we have 40 velocity measurements for each star. The best-fitting orbital solution has $P=0.83935 \pm 0.00002 \mathrm{~d}, K_{1}=123.0 \pm 1.7 \mathrm{~km} \mathrm{~s}^{-1}, K_{2}=92.7 \pm 1.5$ $\mathrm{km} \mathrm{s}^{-1}, \gamma_{1}=-33.6 \pm 1.5 \mathrm{~km} \mathrm{~s}^{-1}$, and a velocity offset of $\gamma_{2}-\gamma_{1}=$ $13.4 \pm 2.5 \mathrm{~km} \mathrm{~s}^{-1}$.

The Lomb-Scargle periodogram shows that the period is well constrained for WD 1606+422. Performing 1000 Monte Carlo simulations, we derive $P=0.83935 \pm 0.00002 \mathrm{~d}, K_{1}=123.0_{-1.8}^{+1.7} \mathrm{~km} \mathrm{~s}^{-1}$,
$K_{2}=92.8 \pm 1.7 \mathrm{~km} \mathrm{~s}^{-1}, \gamma_{1}=-33.4_{-1.9}^{+1.8} \mathrm{~km} \mathrm{~s}^{-1}, \gamma_{2}-\gamma_{1}=13.0_{-3.2}^{+3.0}$ $\mathrm{km} \mathrm{s}^{-1}$, and $\frac{K 1}{K 2}=1.33 \pm 0.03$.

Following our usual procedure, we find that there exists no solution satisfying all available constraints (orbital parameters, spectroscopy, and photometry) simultaneously. However, a consistent solution is achievable if the velocity offset is slightly smaller. Therefore, in what follows, we adopt $\gamma_{2}-\gamma_{1}=11.0 \mathrm{~km} \mathrm{~s}^{-1}$, but keep the original confidence interval for the error propagation. Solving Equation 2 then yields $M_{1}=0.445_{-0.039}^{+0.103} M_{\odot}$ and $M_{2}=0.592_{-0.045}^{+0.124} M_{\odot}$.

Figure 8 displays our best model-atmosphere fit to the spectroscopic and photometric data of WD $1606+422$. As in the case of the other double-lined system WD 0311-649, we assume the surface gravities corresponding to the masses derived from the orbital parameters, $\log g_{1}=7.70_{-0.09}^{+0.19}$ and $\log g_{2}=7.97_{-0.08}^{+0.19}$, while the effective temperatures are determined from the fit. We obtain $T_{\text {eff, } 1}=11,500 \pm 500 \mathrm{~K}$ and $T_{\text {eff, } 2}=13,300 \pm 500 \mathrm{~K}$, for which the Balmer lines and the spectral energy distribution are reproduced well.

Figure 11 compares the observed double $\mathrm{H} \alpha$ feature of WD $1606+422$ with that predicted by our best-fitting solution. As before, we improve the signal-to-noise by co-adding five of our Gemini spectra, and we shift the individual synthetic spectra so that the positions of the observed and theoretical line cores coincide. The agreement is quite good, although our model spectra appear slightly too shallow in the very core of the lines. Finally, similar to WD 0311-649, our analysis suggests that the cooler white dwarf in WD 1606+422 falls within the ZZ Ceti instability strip (Gianninas, Bergeron \& Ruiz 2011). Interestingly enough, Gianninas, Bergeron \& Ruiz (2011) and Bognár, et al. (2018) reported WD 1606+422 to be photometrically constant. However, it is possible that the luminosity variations have not been detected yet because of their dilution by the light of the hotter component. Thus, we recommend that WD $1606+422$ be further monitored for photometric variability.

\section{DISCUSSION}

Bédard, Bergeron \& Fontaine (2017) identified 15 over-luminous white dwarfs in their parallax sample that are inconsistent with being single white dwarfs. Gaia DR2 parallaxes confirm the overluminous nature of all but one of these targets, WD $1130+189$. Out of the 14 remaining binary candidates, one (WD 2048+809) lacks follow-up spectroscopy, four are SB2, four are SB1, and one is a long-period astrometric binary.

Table 1 presents the orbital parameters of all 9 confirmed binary systems in this sample. The four SB2 systems have periods ranging from 0.12 to $1.56 \mathrm{~d}$ with mass ratios of 1.1-1.4, whereas the four SB1 systems have periods in the range $\sim 1-6 \mathrm{~d}$. The newly identified double-lined system WD 0311-649 is almost a twin of the WD 1242-105 binary, though with a much longer orbital period $(0.74 \mathrm{~d}$ versus $0.12 \mathrm{~d})$. WD $1639+153$ is a $P=4$ year astrometric binary detected in ground-based parallax observations by Harris, et al. (2013). Such long-period binary systems are extremely difficult to confirm by radial velocity observations, and are likely hiding in over-luminous white dwarf samples.

Four of the targets in our sample have follow-up radial velocity observations which effectively rule out short-period binary systems. In addition to WD 1418-088 discussed above, WD0126+101, WD0142+312, and WD2111+261 (Maxted et al. 2000; Napiwotzki, et al. 2019) have 8-12 radial velocity observations that do not reveal any significant variability. However, all of these targets appear over-luminous based on their parallax measurements. In addi- 

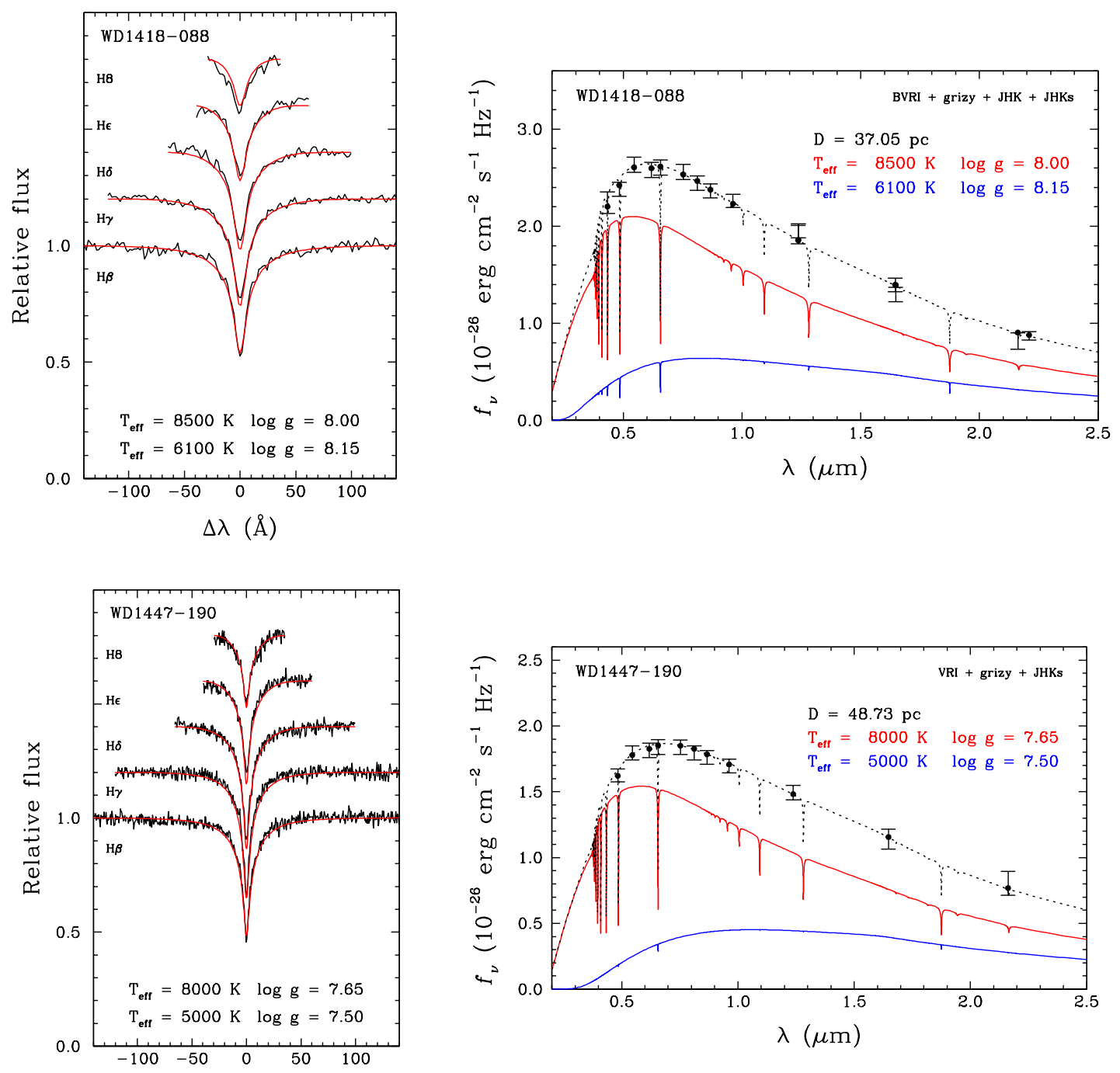

$\Delta \lambda(\AA)$
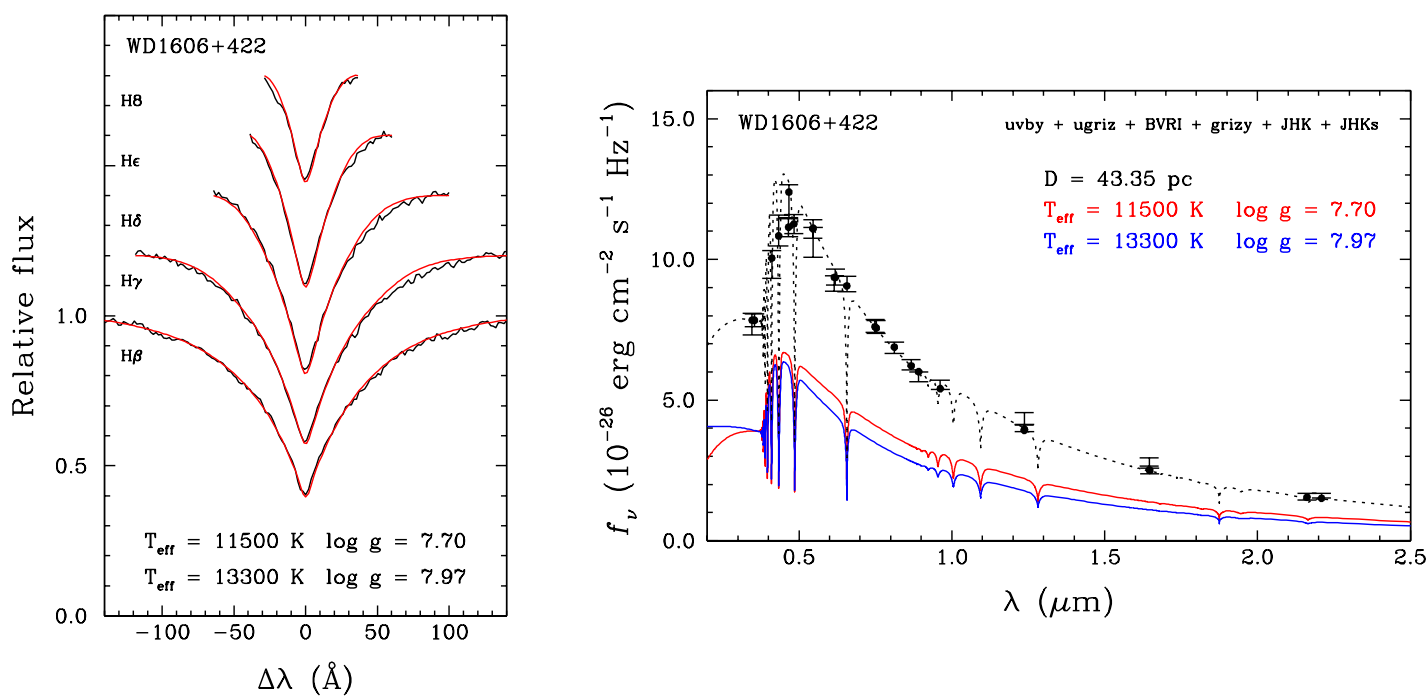

Figure 8. Same as Figure 4, but for WD 1418-088, WD 1447-190, and WD 1606+422. 
Table 1. Orbital and physical parameters of the confirmed binary systems in the Bédard, Bergeron \& Fontaine (2017) sample of over-luminous white dwarfs.

\begin{tabular}{cccccc}
\hline Object & $\varpi$ (mas) & $\mathrm{P}($ days $)$ & Masses $\left(\boldsymbol{M}_{\odot}\right)$ & Type & Reference \\
\hline WD0135-052 & $79.21 \pm 0.04$ & 1.56 & $0.47+0.52$ & SB2 & Saffer, Liebert \& Olszewski (1988) \\
WD0311-649 & $27.29 \pm 0.03$ & 0.74 & $0.39+0.55$ & SB2 & This paper \\
WD1242-105 & $24.80 \pm 0.04$ & 0.12 & $0.39+0.56$ & SB2 & Debes, et al. (2015), Subasavage, et al. (2017) \\
WD1606+422 & $23.07 \pm 0.03$ & 0.84 & $0.45+0.59$ & SB2 & This paper \\
\hline WD0101+048 & $44.86 \pm 0.12$ & $\sim 6.4$ or 1.2 & $0.49+?$ & SB1 & Maxted et al. (2000) \\
WD0326-273 & $43.43 \pm 0.04$ & 1.88 & $0.51+\geqslant 0.59$ & SB1 & Nelemans, et al. (2005) \\
WD1447-190 & $20.52 \pm 0.05$ & 1.79 & $0.41+0.33$ & SB1 & This paper \\
WD1824+040 & $22.42 \pm 0.09$ & 6.27 & $0.43+\geqslant 0.52$ & SB1 & Morales-Rueda, et al. (2005) \\
\hline WD1639+153 & $31.48 \pm 0.11$ & 4 years & $0.93+0.91$ (DA+DA?) & Astrometric & Harris, et al. (2013) \\
& & & $0.98+0.69$ (DA+DC?) & & Harris, et al. (2013) \\
\hline
\end{tabular}
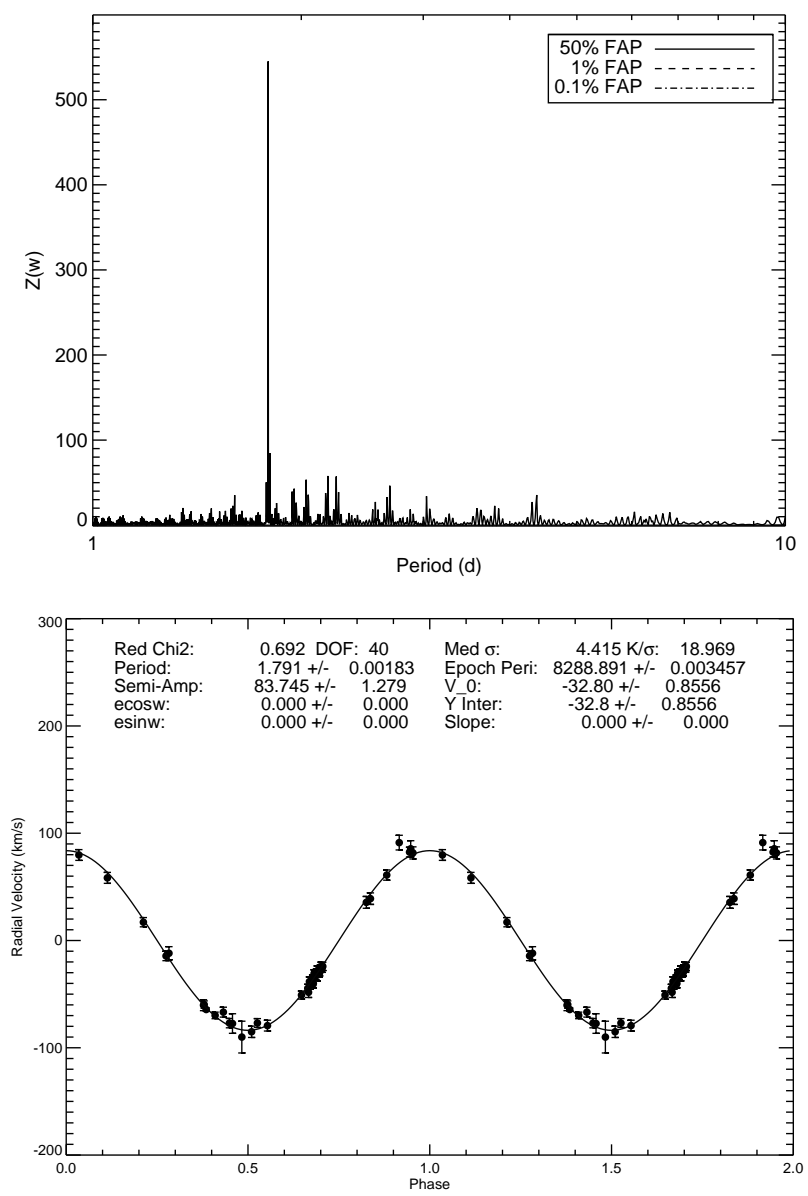

Figure 9. Top: Lomb-Scargle periodogram for WD 1447-190. The period is well constrained and there are no significant period aliases. Bottom: Radial velocity measurements and the best-fitting orbital solution (solid line) for WD 1447-190 assuming a circular orbit. The best-fitting orbital period is $1.79 \mathrm{~d}$.

tion, atmospheric model fits to their spectra suggest average mass white dwarfs, whereas fits to their photometry indicate much lower masses.

For example, WD $0142+312$ has the best-fit spectroscopic estimates of $T_{\text {eff }}=9270 \pm 130 \mathrm{~K}$ and $\log g=8.12 \pm 0.05$ (Limoges, Bergeron \& Lépine 2015), whereas the photometric fit indicates
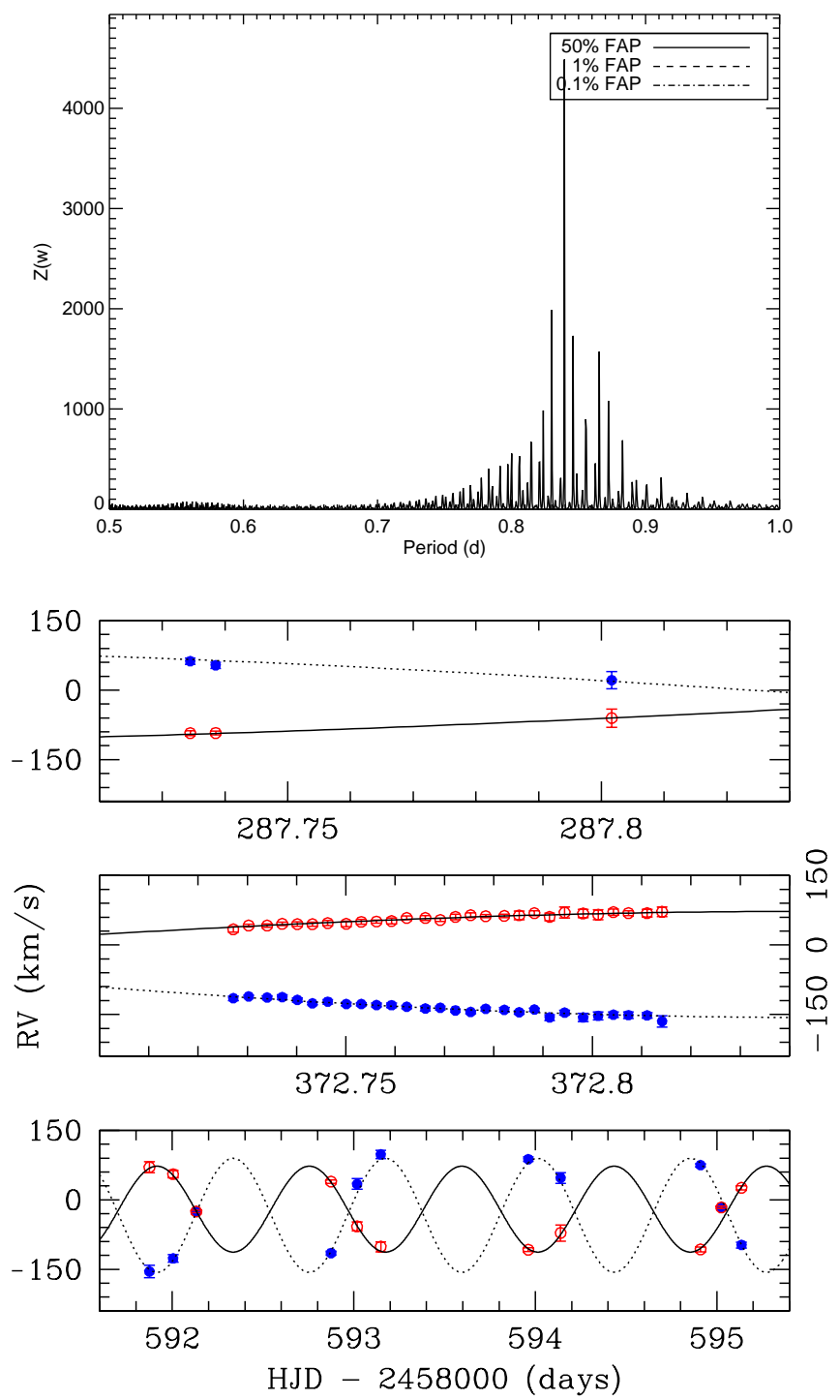

Figure 10. Top: Lomb-Scargle periodogram for WD 1606+422. Bottom: Radial velocity measurements (open and filled points) and the best-fitting orbital solutions (dotted and solid lines) for the two stars in WD 1606+422 assuming a circular orbit. 


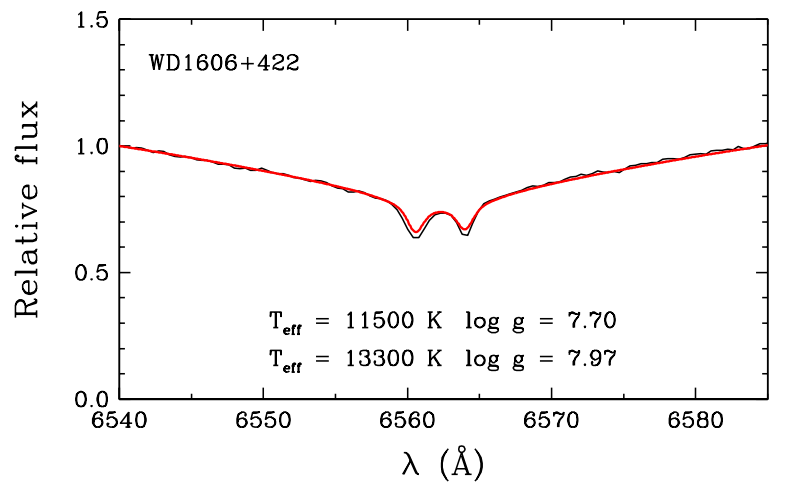

Figure 11. Comparison of the observed double $\mathrm{H} \alpha$ feature of WD 1606+422, shown as the black line, with that predicted by our best model-atmosphere fit, displayed as the red line.

$T_{\text {eff }}=8790 \mathrm{~K}$ and $\log g=7.54$. Both WD $0142+312$ and WD $2111+261$ are inconsistent with being single stars at the $>5 \sigma$ level. The case for a binary system is weaker for WD $0126+101$, as the best-fit estimates from spectroscopy and photometry differ by only $2 \sigma$. Nevertheless, it is clear that at least three (WD $0142+312$, WD 1418-088, and WD 2111+261), and perhaps all four of these objects without any significant radial velocity variations must be binary white dwarfs.

Our observations, as well as the previous radial velocity measurements in the literature, of these targets are not sensitive to month and year-long orbital periods, and would not be able to detect long period systems like the astrometric binary WD 1639+153 (Harris, et al. 2013). Hence, these four targets are likely long period binary systems that can be confirmed through either high-resolution imaging observations or Gaia astrometry (Andrews, Breivik \& Chatterjee 2019).

It is interesting to compare our binary sample to that of the larger sample from the SPY survey. Napiwotzki, et al. (2019) identified 39 double degenerate binaries, half of which are SB2 systems. However, they found significant radial velocity variations in only 16 of the 44 low-mass $\left(M \leqslant 0.45 M_{\odot}\right)$ white dwarfs in their sample, indicating that either these low-mass white dwarfs are single or that they have substellar mass companions. Given that the mean detection efficiency of the SPY survey degrades quickly for month and longer timescales (Napiwotzki, et al. 2019, see their Fig. 6), it is likely that a significant fraction of these are long period binary white dwarfs with higher masses. For example, two of the over-luminous white dwarfs in the Bédard, Bergeron \& Fontaine (2017) sample, WD $0126+101$ and WD 1418-088, are included in the SPY sample as single low-mass white dwarfs, but our analysis suggests that they are instead long period binary systems of more massive white dwarfs.

Figure 12 shows the mass and orbital period distribution of all known double-lined spectroscopic binary white dwarfs and eclipsing double white dwarfs. The lines connect the components of each binary. We limit this figure to only SB2 and eclipsing systems, where the component masses can be constrained reliably. There are 19 SB2 white dwarfs, including the two newly identified systems presented in this work, and 8 eclipsing systems (Hallakoun, et al. 2016; Burdge, et al. 2019, and references therein). The latter are found at short orbital periods ( $P \leqslant 0.25 \mathrm{~d}$ ), and dominated by ELM white dwarfs, which usually have relatively massive companions (Andrews, Price-Whelan \& Agüeros 2014; Boffin 2015; Brown, et

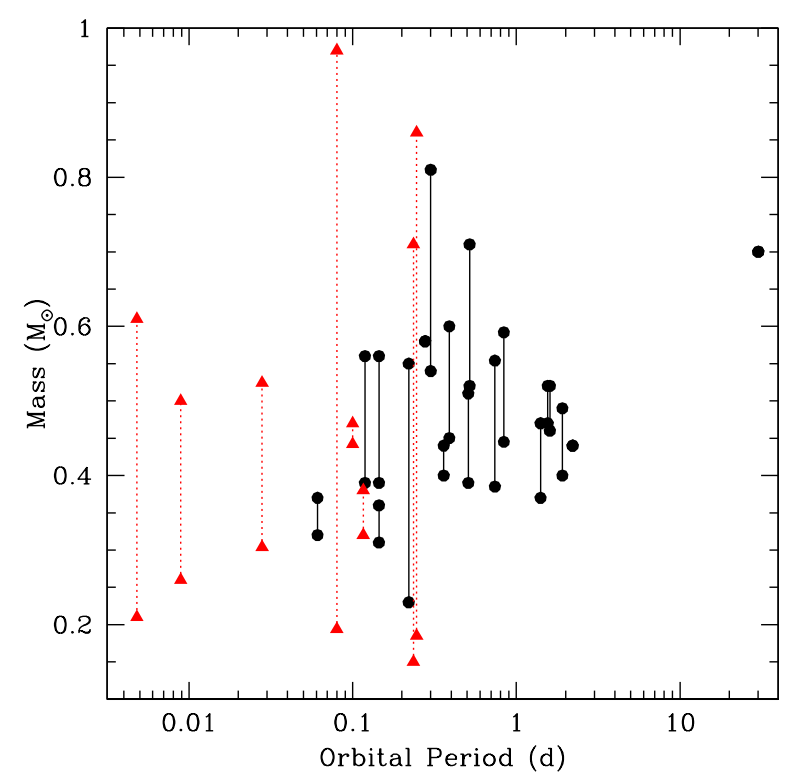

Figure 12. Mass and orbital period distribution of all known SB2 (circles) and eclipsing (triangles) double white dwarfs with orbital constraints. The lines connect the components of each binary.

al. 2017). On the other hand, the double-lined binaries are nearly equal-mass ratio systems. This is not surprising as the spectral lines from both stars would be visible only if both have comparable luminosities, which depend on the radii, and therefore the masses of the two white dwarfs in the system. With further discoveries of doublelined systems among the over-luminous white dwarf population in Gaia (Marsh 2019), we may finally be able to have a large enough sample of SB2 systems to compare against and constrain the population synthesis models for double white dwarfs (e.g., Nelemans, et al. 2001).

\section{CONCLUSIONS}

We presented follow-up spectroscopy of four over-luminous white dwarfs identified by Bédard, Bergeron \& Fontaine (2017). Two of these, WD 0311-649 and WD 1606+422, are double-lined systems, and we provide orbital periods, mass ratios, component masses, and effective temperatures of each binary based on their spectral line profiles, spectral energy distributions, and radial velocity data. An additional system, WD 1447-190, is a single-lined binary with a period of $1.79 \mathrm{~d}$, whereas WD 1418-088 does not show any significant velocity variations.

Studying the 15 over-luminous white dwarfs in the Bédard, Bergeron \& Fontaine (2017) sample, and ignoring WD 2048+809 (no follow-up spectroscopy) and WD 1130+189 (not over-luminous based on Gaia DR2 parallax), we find that four are SB2, four are $\mathrm{SB} 1$, one is an astrometric binary, and four appear to show no significant radial velocity variations. However, there are significant discrepancies between the spectroscopic and photometric fits for the latter four stars, and the only way to resolve this issue is if they are in long period binary systems. Follow-up high spatial resolution imaging and/or Gaia astrometry (Andrews, Breivik \& Chatterjee 2019) may resolve these four systems. We also argue that the overabundance of single low-mass white dwarfs in the SPY survey (Napiwotzki, et al. 2019) is likely due to a similar problem, and 
that at least some of those objects are likely in long period binary systems as well.

Our results provide strong evidence that all 13 of these overluminous white dwarfs with follow-up spectroscopy are indeed double degenerates. In addition, one of these systems, WD 1242-105 has a short enough orbital period to merge within a Hubble time (Debes, et al. 2015). However, we refrain from discussing the implications of our results on the overall space density and merger rate of double white dwarfs due to biases in our sample selection. The 219 stars analyzed in Bédard, Bergeron \& Fontaine (2017) were selected simply because they had a parallax measurement available at the time. They also used parallax measurements from several different sources (e.g., Hipparcos, Yale Parallax Catalog, USNO, Gaia DR1). Hence, our over-luminous white dwarf sample has strong selection effects and is not statistically complete.

\section{ACKNOWLEDGEMENTS}

We thank the referee, Dr. Jeff Andrews, for a constructive report. We gratefully acknowledge the support of the NSF under grant AST1906379. This work is supported in part by the NSERC Canada and by the Fund FRQ-NT (Québec). The authors wish to recognize and acknowledge the very significant cultural role and reverence that the summit of Mauna Kea has always had within the indigenous Hawaiian community. We are most fortunate to have the opportunity to conduct observations from this mountain.

This work benefited from a workshop held at DARK in July 2019 that was funded by the Danish National Research Foundation (DNRF132). We thank Dr. Josiah Schwab for his efforts in organising this workshop.

Based on observations obtained at the Gemini Observatory, which is operated by the Association of Universities for Research in Astronomy, Inc., under a cooperative agreement with the NSF on behalf of the Gemini partnership: the National Science Foundation (United States), National Research Council (Canada), CONICYT (Chile), Ministerio de Ciencia, Tecnología e Innovación Productiva (Argentina), Ministério da Ciência, Tecnologia e Inovação (Brazil), and Korea Astronomy and Space Science Institute (Republic of Korea).

This work was supported by a NASA Keck PI Data Award, administered by the NASA Exoplanet Science Institute. Data presented herein were obtained at the W. M. Keck Observatory from telescope time allocated to the National Aeronautics and Space Administration through the agency's scientific partnership with the California Institute of Technology and the University of California. The Observatory was made possible by the generous financial support of the W. M. Keck Foundation.

Based on observations obtained at the Southern Astrophysical Research (SOAR) telescope, which is a joint project of the Ministério da Ciência, Tecnologia, Inovações e Comunicações (MCTIC) do Brasil, the U.S. National Optical Astronomy Observatory (NOAO), the University of North Carolina at Chapel Hill (UNC), and Michigan State University (MSU).

\section{REFERENCES}

Andrews J. J., Price-Whelan A. M., Agüeros M. A., 2014, ApJL, 797, L32

Andrews J. J., Breivik K., Chatterjee S., 2019, ApJ, 886, 68

Bédard A., Bergeron P., Fontaine G., 2017, ApJ, 848, 11

Bergeron P., et al., 2019, ApJ, 876, 67

Blouin S., Dufour P., Thibeault C., Allard N. F., 2019, ApJ, 878, 63
Boffin H. M. J., 2015, A\&A, 575, L13

Bognár Z., Kalup C., Sódor Á., Charpinet S., Hermes J. J., 2018, MNRAS, 478,2676

Bours M. C. P., et al., 2014, MNRAS, 438, 3399

Brown W. R., Kilic M., Hermes J. J., Allende Prieto C., Kenyon S. J., Winget D. E., 2011, ApJL, 737, L23

Brown W. R., Gianninas A., Kilic M., Kenyon S. J., Allende Prieto C., 2016, ApJ, 818, 155

Brown W. R., Kilic M., Kosakowski A., Gianninas A., 2017, ApJ, 847, 10

Burdge K. B., et al., 2019, Nature, 571, 528

Chambers K. C., et al., 2016, arXiv, arXiv:1612.05560

Clemens J. C., Crain J. A., Anderson R., 2004, SPIE, 331, SPIE.5492

De Lee N., et al., 2013, AJ, 145, 155

Debes J. H., et al., 2015, AJ, 149, 176

Fontaine G., Brassard P., Bergeron P., 2001, PASP, 113, 409

Gaia Collaboration, et al., 2018, A\&A, 616, A1

Gianninas A., Bergeron P., Ruiz M. T., 2011, ApJ, 743, 138

Hallakoun N., et al., 2016, MNRAS, 458, 845

Harris H. C., et al., 2013, ApJ, 779, 21

Holberg J. B., Bergeron P., 2006, AJ, 132, 1221

Karl C. A., Napiwotzki R., Nelemans G., Christlieb N., Koester D., Heber U., Reimers D., 2003, A\&A, 410, 663

Li Z., Chen X., Chen H.-L., Han Z., 2019, ApJ, 871, 148

Limoges M.-M., Bergeron P., Lépine S., 2015, ApJS, 219, 19

Lomb N. R., 1976, Ap\&SS, 39, 447

Marsh T. R., 1995, MNRAS, 275, L1

Marsh T. R., 2019, Close Double White Dwarfs in Gaia

Maxted P. F. L., Ferrario L., Marsh T. R., Wickramasinghe D. T., 2000, MNRAS, 315, L41

Maxted P. F. L., Marsh T. R., Moran C. K. J., 2002, MNRAS, 332, 745

Morales-Rueda L., Marsh T. R., Maxted P. F. L., Nelemans G., Karl C., Napiwotzki R., Moran C. K. J., 2005, MNRAS, 359, 648

Moran C., Marsh T. R., Bragaglia A., 1997, MNRAS, 288, 538

Napiwotzki R., et al., 2001, AN, 322, 411

Napiwotzki R., et al., 2002, A\&A, 386, 957

Napiwotzki R., et al., 2007, ASPC, 387, ASPC..372

Napiwotzki R., et al., 2019, A\&A, in press, arXiv:1906.10977

Nelemans G., Yungelson L. R., Portegies Zwart S. F., Verbunt F., 2001, A\&A, 365, 491

Nelemans G., et al., 2005, A\&A, 440, 1087

Newville M., Stensitzki T., Allen D. B., Ingargiola A., 2014, zndo

Rebassa-Mansergas A., Parsons S. G., García-Berro E., Gänsicke B. T., Schreiber M. R., Rybicka M., Koester D., 2017, MNRAS, 466, 1575

Saffer R. A., Liebert J., Olszewski E. W., 1988, ApJ, 334, 947

Scargle J. D., 1982, ApJ, 263, 835

Steinfadt J. D. R., Kaplan D. L., Shporer A., Bildsten L., Howell S. B., 2010, ApJL, 716, L146

Subasavage J. P., et al., 2017, AJ, 154, 32

Tremblay P.-E., Bergeron P., 2009, ApJ, 696, 1755

Tremblay P.-E., Ludwig H.-G., Steffen M., Freytag B., 2013, A\&A, 559, A104

Vogt S. S., et al., 1994, SPIE, 362, SPIE.2198

This paper has been typeset from a $\mathrm{T}_{\mathrm{E}} \mathrm{X} / \mathrm{L} \mathrm{AT} \mathrm{E} \mathrm{X}$ file prepared by the author.

\section{APPENDIX A: RADIAL VELOCITY DATA}


Table A1. WD 0311-649

\begin{tabular}{|c|c|c|}
\hline $\begin{array}{l}\text { HJD-2450000 } \\
\text { (days) }\end{array}$ & $\begin{array}{r}V 1_{\text {helio }} \\
\left(\mathrm{km} \mathrm{s}^{-1}\right)\end{array}$ & $\begin{array}{l}V 2_{\text {helio }} \\
\left(\mathrm{km} \mathrm{s}^{-1}\right)\end{array}$ \\
\hline 8393.74603027 & $0.0 \pm 3.9$ & $133.7 \pm 4.0$ \\
\hline 8393.74800758 & $-5.3 \pm 7.3$ & $134.8 \pm 4.8$ \\
\hline 8393.75141472 & $-4.2 \pm 6.2$ & $137.1 \pm 5.4$ \\
\hline 8393.75339236 & $3.5 \pm 10.2$ & $133.4 \pm 5.7$ \\
\hline 8393.75536627 & $0.2 \pm 7.7$ & $141.4 \pm 6.4$ \\
\hline 8393.75734092 & $1.3 \pm 7.2$ & $138.3 \pm 5.5$ \\
\hline 8393.75931542 & $5.6 \pm 11.3$ & $135.8 \pm 9.5$ \\
\hline 8393.76129677 & $8.9 \pm 8.2$ & $138.1 \pm 5.3$ \\
\hline 8393.76326492 & $3.3 \pm 7.4$ & $131.4 \pm 5.3$ \\
\hline 8393.76619544 & $-2.6 \pm 7.9$ & $133.0 \pm 5.3$ \\
\hline 8393.76817239 & $7.2 \pm 5.7$ & $135.1 \pm 4.3$ \\
\hline 8393.77014865 & $5.2 \pm 8.1$ & $134.0 \pm 6.2$ \\
\hline 8393.77213876 & $1.6 \pm 8.7$ & $140.4 \pm 7.5$ \\
\hline 8393.77411675 & $11.0 \pm 10.5$ & $140.1 \pm 7.2$ \\
\hline 8393.77608770 & $-0.7 \pm 8.9$ & $125.3 \pm 7.9$ \\
\hline 8393.77806197 & $-3.5 \pm 4.9$ & $128.8 \pm 5.0$ \\
\hline 8393.78099341 & $-4.8 \pm 6.6$ & $129.4 \pm 4.9$ \\
\hline 8393.78297661 & $3.4 \pm 7.0$ & $136.4 \pm 4.9$ \\
\hline 8393.78494521 & $5.5 \pm 7.7$ & $128.0 \pm 5.5$ \\
\hline 8393.78691986 & $10.0 \pm 7.1$ & $133.4 \pm 4.8$ \\
\hline 8393.78889460 & $4.2 \pm 10.9$ & $127.6 \pm 5.1$ \\
\hline 8393.79087339 & $3.6 \pm 8.2$ & $130.9 \pm 6.1$ \\
\hline 8393.79284709 & $10.1 \pm 5.4$ & $128.8 \pm 3.9$ \\
\hline 8393.79576315 & $0.4 \pm 6.8$ & $130.4 \pm 5.8$ \\
\hline 8393.79773732 & $14.9 \pm 12.8$ & $131.9 \pm 8.3$ \\
\hline 8393.79971394 & $13.0 \pm 6.1$ & $132.4 \pm 4.4$ \\
\hline 8393.80169190 & $3.7 \pm 8.8$ & $127.9 \pm 4.9$ \\
\hline 8393.80366792 & $4.5 \pm 7.7$ & $123.9 \pm 5.2$ \\
\hline 8393.80564173 & $7.6 \pm 5.6$ & $120.7 \pm 4.8$ \\
\hline 8393.80761778 & $18.7 \pm 10.3$ & $132.2 \pm 5.3$ \\
\hline 8393.81101586 & $12.4 \pm 6.4$ & $122.1 \pm 5.3$ \\
\hline 8393.81299096 & $8.3 \pm 11.5$ & $122.5 \pm 8.8$ \\
\hline 8393.81496490 & $14.1 \pm 8.5$ & $122.5 \pm 9.6$ \\
\hline 8393.81694324 & $-0.9 \pm 9.2$ & $117.8 \pm 7.3$ \\
\hline 8393.81891741 & $0.4 \pm 8.9$ & $117.4 \pm 6.2$ \\
\hline 8393.82089272 & $4.8 \pm 10.8$ & $115.2 \pm 7.2$ \\
\hline 8393.82286781 & $13.7 \pm 10.0$ & $117.3 \pm 8.3$ \\
\hline 8393.82578447 & $3.3 \pm 9.8$ & $118.3 \pm 6.0$ \\
\hline 8393.82775933 & $5.8 \pm 6.3$ & $119.1 \pm 7.5$ \\
\hline 8393.82973347 & $9.7 \pm 8.5$ & $116.8 \pm 7.3$ \\
\hline 8393.83172374 & $12.0 \pm 11.7$ & $111.9 \pm 7.1$ \\
\hline 8393.83369756 & $10.7 \pm 11.1$ & $104.8 \pm 5.7$ \\
\hline 8393.83568803 & $16.5 \pm 8.7$ & $108.8 \pm 7.1$ \\
\hline 8393.83767901 & $-1.0 \pm 21.2$ & $97.1 \pm 9.7$ \\
\hline 8393.84061334 & $11.5 \pm 17.6$ & $106.2 \pm 12.2$ \\
\hline 8393.84258957 & $15.3 \pm 7.9$ & $103.6 \pm 7.0$ \\
\hline 8393.84456527 & $23.9 \pm 11.4$ & $106.5 \pm 11.1$ \\
\hline 8393.84655678 & $16.4 \pm 20.3$ & $101.5 \pm 12.2$ \\
\hline 8393.84853167 & $11.4 \pm 12.6$ & $97.4 \pm 8.5$ \\
\hline 8393.85050650 & $22.0 \pm 8.5$ & $99.2 \pm 11.6$ \\
\hline 8393.85248162 & $21.3 \pm 12.4$ & $105.2 \pm 13.2$ \\
\hline 8393.85540216 & $5.2 \pm 25.6$ & $92.3 \pm 15.2$ \\
\hline 8393.85737970 & $16.2 \pm 29.0$ & $88.6 \pm 19.3$ \\
\hline 8393.85936985 & $25.3 \pm 12.3$ & $99.8 \pm 11.3$ \\
\hline 8393.86134366 & $11.2 \pm 12.6$ & $84.8 \pm 9.8$ \\
\hline 8393.86333390 & $24.7 \pm 13.2$ & $93.8 \pm 8.9$ \\
\hline 8393.86532509 & $25.3 \pm 13.5$ & $95.5 \pm 12.4$ \\
\hline 8796.50239379 & $114.0 \pm 7.1$ & $-24.3 \pm 7.5$ \\
\hline 8796.64593769 & $56.3 \pm 5.5$ & $56.3 \pm 5.5$ \\
\hline 8796.79867849 & $3.3 \pm 9.7$ & $127.6 \pm 7.9$ \\
\hline 8797.57631070 & $14.1 \pm 9.5$ & $131.7 \pm 8.0$ \\
\hline 8797.67691008 & $56.1 \pm 15.0$ & $97.2 \pm 10.5$ \\
\hline 8797.86212509 & $116.4 \pm 6.6$ & $-25.8 \pm 4.3$ \\
\hline 8798.51407694 & $57.5 \pm 11.4$ & $11.7 \pm 7.6$ \\
\hline 8798.70385557 & $117.1 \pm 8.2$ & $-28.2 \pm 6.4$ \\
\hline 8798.81964492 & $46.5 \pm 3.4$ & $46.5 \pm 3.4$ \\
\hline 8799.51585147 & $96.1 \pm 12.8$ & $-3.3 \pm 13.1$ \\
\hline 8799.75808955 & $1.7 \pm 7.7$ & $129.0 \pm 5.8$ \\
\hline 8799.76176339 & $-1.8 \pm 10.0$ & $135.1 \pm 8.5$ \\
\hline 8801.51700741 & $103.5 \pm 12.3$ & $-10.6 \pm 9.3$ \\
\hline 8801.79271799 & $51.0 \pm 2.2$ & $51.0 \pm 2.2$ \\
\hline
\end{tabular}

Table A2. WD 1418-088

\begin{tabular}{cr}
\hline $\begin{array}{c}\text { HJD-2450000 } \\
\text { (days) }\end{array}$ & $\begin{array}{r}V_{\text {helio }} \\
\left(\mathrm{km} \mathrm{s}^{-1}\right)\end{array}$ \\
\hline 1739.5605 & $-37.1 \pm 1.5$ \\
1742.5722 & $-30.9 \pm 1.6$ \\
8287.75722641 & $-31.3 \pm 2.3$ \\
8287.82388136 & $-28.2 \pm 2.4$ \\
8300.79887603 & $-31.1 \pm 3.9$ \\
8300.80257108 & $-35.1 \pm 4.1$ \\
8300.80700933 & $-28.2 \pm 3.7$ \\
8300.81070462 & $-32.3 \pm 3.1$ \\
8300.81440187 & $-28.8 \pm 4.0$ \\
8300.81809752 & $-34.2 \pm 2.6$ \\
8300.82252975 & $-27.2 \pm 4.8$ \\
8300.82622664 & $-34.2 \pm 3.1$ \\
8300.82992265 & $-27.7 \pm 4.8$ \\
8300.83361871 & $-39.1 \pm 6.0$ \\
8300.83829250 & $-31.1 \pm 4.4$ \\
8300.84198874 & $-37.6 \pm 3.4$ \\
8300.84568688 & $-33.6 \pm 4.2$ \\
8300.84938494 & $-32.6 \pm 4.6$ \\
8300.85382737 & $-36.8 \pm 3.4$ \\
8300.85752113 & $-36.8 \pm 3.0$ \\
8300.86121842 & $-35.1 \pm 3.5$ \\
8300.86491463 & $-28.6 \pm 3.9$ \\
8300.86934108 & $-34.6 \pm 4.3$ \\
8300.87303809 & $-33.2 \pm 4.3$ \\
\hline &
\end{tabular}




\section{Kilic et al.}

Table A3. WD 1447-190

\begin{tabular}{|c|c|}
\hline $\begin{array}{c}\text { HJD-2450000 } \\
\text { (days) }\end{array}$ & $\begin{array}{r}\text { Vhelio } \\
\left(\mathrm{km} \mathrm{s}^{-1}\right)\end{array}$ \\
\hline 8287.79004386 & $-97.1 \pm 1.5$ \\
\hline 8287.83398725 & $-102.6 \pm 3.1$ \\
\hline 8309.78038901 & $-77.4 \pm 3.8$ \\
\hline 8309.78408475 & $-81.1 \pm 4.8$ \\
\hline 8309.78851329 & $-71.1 \pm 4.4$ \\
\hline 8309.79220918 & $-74.4 \pm 3.9$ \\
\hline 8309.79590724 & $-71.6 \pm 4.6$ \\
\hline 8309.79960426 & $-69.4 \pm 2.6$ \\
\hline 8309.80402782 & $-70.0 \pm 4.9$ \\
\hline 8309.80771825 & $-72.9 \pm 4.1$ \\
\hline 8309.81141411 & $-63.6 \pm 3.4$ \\
\hline 8309.81511482 & $-66.0 \pm 4.0$ \\
\hline 8309.81953192 & $-65.9 \pm 3.2$ \\
\hline 8309.82322608 & $-63.0 \pm 3.8$ \\
\hline 8309.82692321 & $-63.5 \pm 7.0$ \\
\hline 8309.83062011 & $-60.4 \pm 4.0$ \\
\hline 8309.83504889 & $-63.4 \pm 3.7$ \\
\hline 8309.83874442 & $-62.9 \pm 2.9$ \\
\hline 8309.84244060 & $-58.3 \pm 2.8$ \\
\hline 8309.84613589 & $-57.4 \pm 4.5$ \\
\hline 8309.85055716 & $-58.7 \pm 3.8$ \\
\hline 8309.85425293 & $-57.0 \pm 3.8$ \\
\hline 8544.82924001 & $58.4 \pm 6.8$ \\
\hline 8544.88585415 & $52.7 \pm 7.5$ \\
\hline 8545.75376632 & $-99.6 \pm 4.6$ \\
\hline 8545.79880531 & $-110.2 \pm 9.1$ \\
\hline 8545.84483738 & $-122.8 \pm 14.8$ \\
\hline 8545.89278086 & $-117.9 \pm 5.3$ \\
\hline 8546.83294299 & $46.9 \pm 5.0$ \\
\hline 8588.63922836 & $-92.5 \pm 3.4$ \\
\hline 8588.76353703 & $-109.8 \pm 4.4$ \\
\hline 8588.90132700 & $-109.7 \pm 4.1$ \\
\hline 8602.77792314 & $-47.2 \pm 4.4$ \\
\hline 8602.79230148 & $-44.7 \pm 6.1$ \\
\hline 8603.86506466 & $28.2 \pm 4.8$ \\
\hline 8605.55550400 & $2.8 \pm 5.5$ \\
\hline 8605.76824108 & $49.6 \pm 4.5$ \\
\hline 8606.54405099 & $-93.1 \pm 5.1$ \\
\hline 8606.85924347 & $-112.1 \pm 5.0$ \\
\hline 8616.52990044 & $48.9 \pm 5.6$ \\
\hline 8616.81562358 & $25.6 \pm 5.1$ \\
\hline 8636.69212753 & $-15.7 \pm 4.3$ \\
\hline 8637.47080183 & $-83.7 \pm 3.7$ \\
\hline 8637.81049184 & $6.2 \pm 5.4$ \\
\hline
\end{tabular}

Table A4. WD 1606+422

\begin{tabular}{rrr}
\hline $\begin{array}{r}\text { HJD-2450000 } \\
(\text { days })\end{array}$ & $\begin{array}{r}V 1 \text { helio } \\
\left(\mathrm{km} \mathrm{s}^{-1}\right)\end{array}$ & $\begin{array}{r}\text { V2 helio } \\
\left(\mathrm{km} \mathrm{s}^{-1}\right)\end{array}$ \\
\hline 8287.73442998 & $62.1 \pm 6.7$ & $-92.9 \pm 5.4$ \\
8287.73849062 & $53.7 \pm 6.7$ & $-92.6 \pm 4.2$ \\
8287.80162519 & $21.6 \pm 18.4$ & $-60.4 \pm 19.5$ \\
8372.72714356 & $-115.1 \pm 4.9$ & $33.9 \pm 6.0$ \\
8372.73020305 & $-111.2 \pm 3.0$ & $41.7 \pm 2.9$ \\
8372.73398796 & $-113.2 \pm 3.1$ & $41.8 \pm 3.8$ \\
8372.73704803 & $-112.7 \pm 5.2$ & $45.7 \pm 3.8$ \\
8372.74011007 & $-118.4 \pm 4.4$ & $45.0 \pm 4.8$ \\
8372.74316907 & $-126.0 \pm 3.0$ & $45.1 \pm 4.4$ \\
8372.74623077 & $-122.7 \pm 5.1$ & $47.3 \pm 5.7$ \\
8372.75003536 & $-127.5 \pm 4.0$ & $45.6 \pm 5.9$ \\
8372.75309717 & $-127.8 \pm 3.2$ & $49.8 \pm 5.1$ \\
8372.75615667 & $-129.7 \pm 4.0$ & $50.4 \pm 3.3$ \\
8372.75921767 & $-130.2 \pm 4.7$ & $51.7 \pm 6.4$ \\
8372.76227795 & $-133.7 \pm 3.4$ & $58.4 \pm 4.1$ \\
8372.76606043 & $-137.4 \pm 5.0$ & $58.2 \pm 4.9$ \\
8372.76911994 & $-136.0 \pm 4.3$ & $54.0 \pm 3.3$ \\
8372.77217918 & $-141.4 \pm 3.1$ & $60.3 \pm 6.1$ \\
8372.77524041 & $-144.9 \pm 3.5$ & $64.0 \pm 5.0$ \\
8372.77830567 & $-138.1 \pm 4.2$ & $62.2 \pm 3.6$ \\
8372.78208815 & $-140.1 \pm 4.7$ & $62.7 \pm 3.9$ \\
8372.78514742 & $-145.3 \pm 3.9$ & $63.9 \pm 9.5$ \\
8372.78820890 & $-139.6 \pm 3.3$ & $68.4 \pm 5.2$ \\
8372.79126849 & $-156.7 \pm 6.3$ & $60.3 \pm 8.1$ \\
8372.79432937 & $-146.1 \pm 5.0$ & $70.0 \pm 11.8$ \\
8372.79810607 & $-156.5 \pm 8.6$ & $67.9 \pm 8.6$ \\
8372.80116626 & $-153.1 \pm 8.8$ & $65.3 \pm 10.6$ \\
8372.80422631 & $-150.8 \pm 5.8$ & $70.7 \pm 6.6$ \\
8372.80728686 & $-151.7 \pm 7.0$ & $68.7 \pm 5.3$ \\
8372.81106865 & $-152.2 \pm 6.1$ & $68.7 \pm 7.9$ \\
8372.81412870 & $-165.0 \pm 11.9$ & $71.5 \pm 10.6$ \\
8591.87407156 & $-154.6 \pm 13.5$ & $70.2 \pm 12.0$ \\
8592.00464574 & $-126.5 \pm 8.1$ & $55.2 \pm 7.8$ \\
8592.13206901 & $-25.2 \pm 2.8$ & $-25.2 \pm 2.8$ \\
8592.87443003 & $-115.1 \pm 3.9$ & $39.0 \pm 3.5$ \\
8593.01822250 & $34.1 \pm 11.5$ & $-57.6 \pm 9.6$ \\
8593.14855262 & $97.7 \pm 8.9$ & $-101.3 \pm 10.8$ \\
8593.96107311 & $87.5 \pm 4.8$ & $-107.7 \pm 4.2$ \\
8594.13898214 & $47.1 \pm 11.7$ & $-71.8 \pm 17.4$ \\
8594.90966600 & $74.8 \pm 3.3$ & $-107.2 \pm 5.3$ \\
8595.02447094 & $-16.3 \pm 2.4$ & $-16.3 \pm 2.4$ \\
8595.13547162 & $-97.8 \pm 6.4$ & $25.7 \pm 4.6$ \\
\hline & &
\end{tabular}

